\title{
Systematisierung, Bewertung und Modellierung der Unsicherheiten in der Leercontainerlogistik
}

\author{
Systematization, Evaluation, and Modelling of Uncertainties \\ in Empty Container Logistics
}

\author{
Nina Vojdani \\ René Rösner \\ Lehrstuhl für Produktionsorganisation und Logistik \\ Fakultät für Maschinenbau und Schiffstechnik \\ Universität Rostock
}

D ieser Beitrag beschreibt Unsicherheiten in den Prozessen der Leercontainerlogistik und beinhaltet einen Systematisierungsansatz, der die Akteure bei der operativen Planung unterstützen soll. Weiterhin werden ausgewählte Modellierungskonzepte zur Berücksichtigung von Unsicherheiten vorgestellt und hinsichtlich ihrer Eignung zum Einsatz in mathematischen Optimierungsmodellen für das Leercontainermanagement analysiert. An einem konkreten Fallbeispiel wird der mögliche Einbezug der sogenannten Grey-Zahlen verdeutlicht.

[Schlüsselwörter: Leercontainerlogistik, Unsicherheiten, Fuzzy, Grey-Zahlen, Operations Research]

$\mathbf{T}$ his paper analyzes uncertainties in empty container logistics and includes a systematization approach that should support actors in operative planning. Furthermore, selected methods to model uncertainties are presented and analyzed in terms of their appropriateness for the empty container management using mathematical optimization models. A case study illustrates the application of grey numbers.

[Keywords: empty container logistics, uncertainties, fuzzy, grey numbers, operations research]

\section{EINFÜHRUNG}

Die globale Vernetzung der Wirtschaft in der heutigen Form wäre ohne den Einsatz von Containern nicht umsetzbar. Der Erfolg der Container ist das Resultat der Zusammenführung unzähliger Güterformen zu einer kleinen Menge von standardisierten Einheiten [OZF05]. Mit der Vereinheitlichung der Transportbehälter mit internationalen Standardmaßen geht eine Vielzahl von Vorteilen wie z. B. kürzere Umschlagszeiten einher. Neben positiven Effekten ist jedoch auch die Problematik der Repositionierung leerer Container entstanden. Auf jeden Vollcontainertransport muss in der Regel ein Transport des leeren Containers zu seinem neuen Bestimmungsort erfol- gen. Das heißt, durch den Vollcontainerfluss wird ein Leercontainerfluss induziert [Voj10]. Die dafür benötigten Prozess- und Kapazitätsaufwendungen sind aus ökonomischen, sozialen und ökologischen Gründen zwingend zu reduzieren.

Als wichtigste Quelle für Leercontainertransporte und -anhäufungen gelten unpaarige Handelsströme, ein strukturelles Problem, mit dem sich die globale Wirtschaft in der Seeschifffahrt konfrontiert sieht. Die Gestaltung optimaler Leercontainerströme und -bestände hat sich in den letzten beiden Dekaden als eine der größten Managementaufgaben der Containerbesitzer verfestigt. So stuften in einer aktuellen Umfrage unter 76 Akteuren der Containerlogistik 71 Prozent der Befragten das Thema Leercontainerlogistik als ,sehr wichtig“ und 26 Prozent als „eher wichtig“ ein [Hüt12].

In der Literatur befinden sich zahlreiche Ansätze zur Reduzierung der Transport-, Umschlags- und Lagerprozesse von leeren Containern. Neben organisatorischen und technischen Ansätzen sind insbesondere mathematische Modelle entwickelt worden, um das Leercontainermanagement zu unterstützen. Die Umsetzung der mathematischen Optimierungsverfahren von der Theorie in die Praxis ist jedoch nicht immer entsprechend möglich. Hauptgründe hierfür sind Unsicherheiten und unvollständige Informationen in den Planungsprozessen, die in theoretischen Betrachtungen nur bedingt abgebildet werden. Beispielsweise kann ein Container, der nach Planung zum Transport neuer Waren bereitstehen soll, aufgrund unerwarteter Verspätungen bei der Rückgabe durch den vorherigen Importeur nicht vorhanden sein. Weiterhin geben Reedereien an, dass der Aufwand für die exakte Datenbeschaffung teilweise den Nutzen übersteigt.

Insbesondere die Anwendbarkeit deterministischer Verfahren wird durch stochastische Einflüsse und unvollständige Informationen beträchtlich gemindert. Auch wenn der Einsatz deterministischer Optimierungsmodelle die Repositionierung von Leercontainern gegenüber heuristischen und dezentralen Vorgehensweisen in der Praxis 
verbessert, bleiben aufgrund des komplexen globalen Systems der Containerlogistik und den daraus entstehenden zahlreichen unberücksichtigten bzw. ungewissen Aspekten weitere Optimierungspotentiale vorhanden. Diese Potentiale sind angesichts der enormen Einsparungsmöglichkeiten, die mit einem optimalen Management der Leercontainerflüsse einhergehen, weiter zu nutzen.

Ziel dieses Beitrags ist es, einen Ansatz zur Systematisierung der Unsicherheiten der maritimen Leercontainerlogistik aufzuzeigen und bzgl. ihres Einflusses auf die operative Planung und Optimierung der Containerlogistik zu bewerten. Insbesondere soll die Modellierung der von Unsicherheiten geprägten Parameter als sogenannte Greyund Fuzzy-Variablen untersucht werden. Diese Modellierungsansätze erlauben es, Unsicherheiten bzw. unscharfe Aussagen der Praxis in mathematischen Modellierungsansätzen zu berücksichtigen und eine praxisgerechte Optimierung zu ermöglichen.

Die Ergebnisse sollen zum einen die Akteure der maritimen Containerlogistik in ihren Planungsprozessen unterstützen, indem Zusammenhänge und Auswirkungen besser beurteilt werden können und somit verbesserte Entscheidungen bei nicht perfektem Wissen ermöglichen. Zum anderen sollen die Resultate die Grundlage für verbesserte Optimierungsverfahren bilden, die Unsicherheiten angemessen berücksichtigen können.

Im Folgenden wird zunächst der Begriff Unsicherheit diskutiert sowie ausgewählte Modellierungskonzepte vorgestellt. Das Kapitel 3 beinhaltet eine Übersicht wichtiger Publikationen zum Thema Leercontainermanagement, deren Optimierungsmodelle Unsicherheiten berücksichtigen. Im Anschluss erfolgt die Erläuterung eines prozessorientierten Ansatzes zur Systematisierung der Unsicherheiten in der Planung von Leercontainerströmen. Den Abschluss bildet ein Fallbeispiel zur Anwendung des sogenannten Grey-Modellierungskonzepts in linearen Optimierungsmodellen auf Grundlage eines Netzwerkansatzes.

\section{UNSICHERHEITEN UND MODELLIERUNGSKONZEPTE}

Allgemein können die Arten und Ursachen von Unsicherheiten sehr verschieden sein. Abhängig von diesen Ausprägungen eignen sich unterschiedliche Modellierungskonzepte. Vor Behandlung der Unsicherheiten der Leercontainerlogistik sind eine kurze allgemeine Einführung und eine Festlegung der Terminologie daher sinnvoll.

\subsection{Der BegrifF UNSICHERHEIT}

In der Literatur existiert eine Vielzahl von Systematisierungen im Bezug auf den Begriff Unsicherheiten. Die wichtigsten Arten der Unsicherheiten sollen mit den folgenden Definitionen abgedeckt werden. Ein bekannter Ansatz zur Differenzierung des Begriffs Unsicherheit ist die Einteilung in aleatorische und epistemische Unsicherheit [HJO10].

Aleatorische Unsicherheiten leiten sich von inhärentem Zufall eines betrachteten Systems ab und resultieren nicht aus einem quantitativen Mangel an Informationen. Ein Beispiel hierfür wäre die Unsicherheit über einen Stau aufgrund eines Unfalls. Selbst bei beliebig exakter Informationslage besteht keine absolute Sicherheit über Zeitpunkt und Ort dieses Ereignisses. Epistemische Unsicherheit folgt aus einem Mangel an exakten Informationen. Bei unzureichender Informationsverwaltung ist einem Entscheidungsträger z. B. nicht bekannt, wie viele Container sich zum gegenwärtigen Zeitpunkt in einem bestimmten Depot befinden, so dass eine subjektive Beurteilung heranzuziehen ist. Eine Reduzierung von Unsicherheiten epistemischer Art ist durch Informationsbeschaffung möglich, aber teilweise sehr aufwändig und eventuell nicht sinnvoll.

Für epistemische Unsicherheiten lässt sich das vorhandene Wissen durch drei Eigenschaften charakterisieren [BK01]. Eine Information ist unpräzise, wenn statt eines genauen Zahlenwerts ein gewisser numerischer Bereich bekannt ist (,drei bis sechs Container"), aber keine weitere Aussage über diesen Bereich möglich bzw. verfügbar ist. Eine Information wird als ungewiss bezeichnet, wenn der reale Wert von der verfügbaren Information abweichen kann (im Depot sind sieben, statt „drei bis sechs" Container). Die Information wird als vage bezeichnet, wenn aus ihr kein numerischer Wert mit Sicherheit gefolgert werden kann. Wird z. B. angegeben, dass in einem Depot „viele“ Container zur Verfügung stehen, ist eine genaue Quantifizierung im Allgemeinen nicht möglich.

Die Ursachen für die Unsicherheiten können verschiedener Natur sein und sollten einem Entscheidungsträger bewusst sein. Auf Basis von Zimmermann lassen sich fünf relevante Ursachen unterscheiden [Zim00].

- Informationsmangel: Die wohl häufigste Ursache ist das nicht Vorhandensein jeglicher Information über ein Ereignis.

- Informationskomplexität: Ist ein Entscheidungsträger aufgrund eines sehr komplexen Systems mit einer Informationsflut konfrontiert, kann eine Filterung der Daten zu Unsicherheiten führen.

- Informationskonflikt: Für einen Sachverhalt können widersprüchliche Informationen zur Verfügung stehen, beispielsweise aufgrund verschiedener Expertenaussagen.

- Informationsmehrdeutigkeit: Gleiche linguistische Informationen können unterschiedliche numerische Angaben meinen. 
- Subjektivität: Vorliegende Informationen sind unsicher, wenn sie auf subjektiven Einschätzungen basieren.

\subsection{MODELLIERUNGSKONZEPTE}

Für die Repräsentation von Unsicherheiten sind zahlreiche Modellierungskonzepte entwickelt worden. In diesem Abschnitt soll eine kurze Vorstellung der Wahrscheinlichkeits-, Fuzzy-Mengen- und Grey-ZahlenTheorie erfolgen. Als weitere bekannte Konzepte seien die Possibility Theory [Zad78], Dempster-ShaferEvidenztheorie [Gle76] sowie die Grobmengentheorie [Paw82] genannt.

\subsubsection{WAHRSCHEINLICHKEITSTHEORIE}

Die Wahrscheinlichkeitstheorie ist ein Teilgebiet der Stochastik. Sie basiert auf der Definition von Wahrscheinlichkeitsräumen der Form $(\Omega, \Sigma, \mathrm{P})$. Ereignisse werden als Teilmengen einer Ergebnismenge $\Omega$ aufgefasst. Um Systemen von Mengen Wahrscheinlichkeiten zuordnen zu können, wird ein Mengensystem, die sogenannte $\Sigma$ Algebra, gebildet. Diese erfüllt nach Definition folgende drei Eigenschaften: $\Omega \in \Sigma$; wenn $A \in \Sigma$, dann ist das Komplement $\Omega \backslash A \in \Sigma$; wenn die Mengen $\mathrm{A}_{1}, \mathrm{~A}_{2}, \ldots \in \Sigma$, dann ist auch die Vereinigung $\bigcup_{i=1}^{\infty} A_{i} \in \Sigma$. Weiterhin existiert eine Funktion $\mathrm{P}: \Sigma \longrightarrow[0,1]$, das Wahrscheinlichkeitsmaß, das nach den Axiomen von Kolmogorow folgende Eigenschaften besitzt:

- $\mathrm{A} \in \Omega \Rightarrow \mathrm{P}(\mathrm{A}) \in[0,1]$

- $\mathrm{P}(\Omega)=1$

- $\quad \mathrm{P}\left(\mathrm{U}_{\mathrm{i}=1}^{\mathrm{n}} \mathrm{A}_{\mathrm{i}}\right)=\sum_{\mathrm{i}=1}^{\mathrm{n}} \mathrm{P}\left(\mathrm{A}_{\mathrm{i}}\right) \forall \mathrm{A}_{\mathrm{i}} \cap \mathrm{A}_{\mathrm{j}}=\varnothing, \forall \mathrm{i} \neq \mathrm{j}$

Einem Ereignis $\omega \in \Omega$ wird auf Basis dieser theoretischen Grundlagen genau eine Wahrscheinlichkeit $\mathrm{P}(\{\omega\})$ zugeordnet, im Gegensatz zu anderen Modellierungskonzepten wie der Evidenz- oder Possibilitytheorie [HJO10].

Eine messbare Funktion $\mathrm{X}: \Omega \rightarrow \mathbb{R}$ wird als reelle $\mathrm{Zu}$ fallsvariable bezeichnet. Diese besitzt auf Grundlage des Wahrscheinlichkeitsmaßes $\mathrm{P}$ eine Wahrscheinlichkeitsverteilung $\mathrm{P}_{\mathrm{X}}=\mathrm{P} \circ \mathrm{X}^{-1}$. Zufallsvariablen können reale Ge- gebenheiten unter stochastischen Einflüssen abbilden und besitzen im Allgemeinen Charakteristika wie Erwartungswert und Varianz.

\subsubsection{GREY-MENGEN-THEORIE}

Für eine Greyzahl $\mathrm{g}$ gilt: $\mathrm{g}=\left[\mathrm{g}^{-}, \mathrm{g}^{+}\right]$mit $\mathrm{g}^{-}, \mathrm{g}^{+} \in \mathrm{R} \cup\{-\infty, \infty\}$ und $\mathrm{g}^{-} \leq \mathrm{g}^{+}$. Eine Greyzahl ist dementsprechend ein Intervall und modelliert einen Zahlenbereich, über den keine genaueren Informationen über die Wahrscheinlichkeitsverteilung bekannt sind. Es kann nur sicher gesagt werden, dass der zu modellierende Wert zwischen den Intervallgrenzen liegt. Auf Basis dieser Theorie ist die Integration von Unsicherheiten unter anderem in speziell entwickelten Optimierungsverfahren möglich [Ros09].

\subsubsection{FUZZY-MENGEN-THEORIE}

In der klassischen Mengentheorie wird im Hinblick auf die Zugehörigkeit von Elementen zu einer Menge nur zwischen zwei Fällen unterschieden: das Element ist enthalten oder nicht enthalten. Zur Erweiterung dieser Logik wurde von Zadeh die Fuzzy-Logik eingeführt [Zad65]. In der Fuzzy-Theorie werden Elemente auf Basis einer $\mathrm{Zu}-$ gehörigkeitsfunktion einer Menge zugeordnet. Es wird somit unterschieden, zu welchem Grad eine Menge ein bestimmtes Element enthält. Mit diesem Konzept lassen sich insbesondere unscharfe Aussagen modellieren. Im Fall der Leercontainerlogistik könnte z. B. eine Aussage wie „der Leercontainerbedarf wird in einer Woche sehr hoch" sein, als unscharfe Menge aufgefasst werden. Numerische Angaben können nun zu einem gewissen Grad dieser Menge zugeordnet werden. Etwa: Zehn Container gehören mit einem Grad von 0,1 zur Menge, 20 Container mit einem Grad von 0,9 .

\subsection{BEWERTUNG DER ANWENDUNG DER MODELLIERUNGSKONZEPTE}

Die Wahl der bestmöglichen Modellierungsmethode hängt von Ursache und Art der Unsicherheiten, aber auch von anderen Aspekten wie benötigte Informationsdarstellung ab [Zim99]. Im Folgenden wird ein Ansatz zur Auswahl beschrieben, der in Abbildung 1 dargestellt ist.

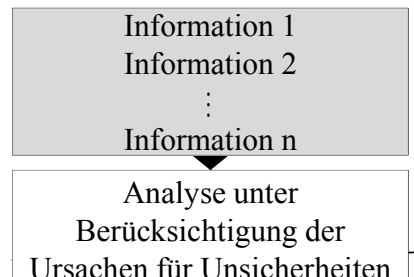

Ursachen für Unsicherheiten

\begin{tabular}{|c|} 
Verdichtung der Informationen \\
zu Inputparametern für \\
Optimierungsmodell
\end{tabular}

Identifizierung der Unsicherheitsart
Auswahl des geeigneten Modellierungskonzepts

Inputdaten mit Modellierungskonzept für das Optimierungsmodell

Abbildung 1. Bestimmung des Modellierungskonzepts 
Zunächst müssen die verfügbaren Informationen unter Berücksichtigung der genannten Ursachen für Unsicherheiten analysiert werden. Anschließend sind die Informationen $\mathrm{zu}$ Inputparametern für ein gewähltes Optimierungsmodell zu verdichten, wobei die Art der Unsicherheit jeweils $\mathrm{zu}$ bestimmen ist. Je nach Unsicherheitsart werden geeignete Modellierungsmethoden ausgewählt. Eine Veranschaulichung dieses Prozesses befindet sich in Abbildung 1.

Allgemein sind für aleatorische Unsicherheiten $\mathrm{Zu}$ fallsvariablen mit entsprechender Wahrscheinlichkeitsverteilung geeignet [HJO10]. Bei vagen Parametern sollte die Modellierung mit Hilfe von Fuzzy-Mengen erfolgen, um unscharfe Aussagen berücksichtigen zu können. Für sichere unpräzise Parameter eignet sich die Modellierung mit Grey-Zahlen, während für ungewisse unpräzise Parameter wiederum eine Fuzzy-Modellierung ein geeigneter Ansatz sein kann. Diese Bewertung ist nicht allgemeingültig und hängt insbesondere von der Anwendung in der Praxis bzw. des späteren Optimierungsmodells ab.

\section{LITERATURANALYSE}

Die Veröffentlichungen zur mathematischen Optimierung des Leercontainermanagements gliedern sich hauptsächlich in zwei Kategorien - deterministische und stochastische Ansätze. Deterministische Ansätze ([EKP72; SK95; EMS05; OZF05] etc.) basieren auf der Annahme, dass alle Inputparameter exakt einen Wert annehmen und lassen die Betrachtung von Unsicherheiten nur durch Integration von Erwartungswerten zu. Negative Auswirkungen auf die Optimallösung durch auftretende Unsicherheiten können nur durch Anwendung der „Rolling Horizon"-Methode reduziert werden. Hierbei wird die Optimierung zu jeder Planungsperiode auf Basis der aktuell verfügbaren Daten durchgeführt.

Unter anderem zeigen Cheung und Chen, dass ein stochastischer Optimierungsansatz Kosteneinsparungspotential gegenüber deterministischen Verfahren besitzt [CC98]. Die Bedeutung der Modellierung und Algorithmenentwicklung unter expliziter Berücksichtigung unsicherer Einflüsse haben in der Folge zugenommen. Die betrachteten unsicheren Parameter und deren Modellierung unterschieden sich jedoch selten. Eine Auswahl sei im Folgenden vorgestellt.

Crainic et al. entwickelten im Rahmen der Untersuchung von Inlandstransporten für Leercontainer sowohl ein deterministisches als auch ein stochastisches Modell [CGD93]. In diesem wurden die zwei Einflussgrößen Leercontainerbedarf und -angebot als unsicher betrachtet. Ein zweistufiges stochastisches Optimierungsmodell wurde von Cheung und Chen entwickelt [CC98]. Während in der ersten Phase alle Daten deterministisch sind, werden Leercontainerangebot, -bedarf und auch Transportkapazität in der zweiten Phase als Zufallsvariablen definiert. Ein stochastisches Optimierungsproblem mit unsicherer Nachfrage und Bestand für die Planung von Leercontainerbewegungen in einer Periode wurde von Bean und Joubert präsentiert [BJ10].

Epstein et al. beschreiben die Implementierung eines Systems zur Optimierung der Leercontainerprozesse bei der global operierenden südamerikanischen Reederei CSAV [ENW12]. Verfügbare und nachgefragte Leercontainermengen sowie Ankunftszeiten wurden als unsicher berücksichtigt. Die Transportkapazität von Containerschiffen wurde über einen speziellen Faktor im Bestandsmanagement der Depots integriert. Die unsicheren Gegebenheiten des Leercontainermanagements wurden ebenfalls von Long et al. untersucht [LLC12]. Ein zweistufiges stochastisches Optimierungsmodell mit unsicheren Ein- und Ausgängen von Leercontainern sowie freien Plätzen auf Containerschiffen wurde entwickelt. Insbesondere fand auch die Restriktion von unsicheren Gewichtsbeschränkungen bei Containerschiffen Berücksichtigung im Modell. Alle bisher genannten Publikationen berücksichtigen Unsicherheiten nur mit Hilfe von Zufallsvariablen, die Betrachtungen auf Basis der Wahrscheinlichkeitstheorie zulassen. Andere Ansätze zur Modellierung von Unsicherheiten sind weniger verbreitet.

Di Francesco et al. beschreiben das Problem der Annahme, zuverlässige Datenbestände, die den Einsatz geeigneter Wahrscheinlichkeitsverteilung für Zufallsvariablen erlauben, seien immer verfügbar [DCZ08]. Um diese Annahme zu vermeiden, erfolgte die Entwicklung eines Multi-Szenario-Ansatzes zur Repositionierung von leeren Containern. Die Bildung der Szenarien soll dabei auf Basis von subjektiven Expertenaussagen erfolgen. Erera et al. entwickelten auf Basis eines Raum-Zeit-Netzwerks einen Ansatz zur robusten Optimierung für das Leercontainerproblem. Eingehende Leercontainer wurden mit einer intervallbasierten Methode als unsicher integriert [EMS07]. Den Einsatz eines Mixed-Fuzzy-Modells wählen Chou et al. [CGT10]. In ihrem Beitrag wird zunächst der optimale Leercontainerbestand in den betrachteten Regionen auf Basis von Fuzzy-Angaben für Bestellmengen, Lager-, Bestellkosten etc. bestimmt, um danach mit Hilfe eines deterministischen Netzwerkansatzes die optimalen Repositionierungsmengen festzulegen.

Eine gezielte Analyse von Unsicherheiten in der Leercontainerlogistik, die eine Basis für die Bewertung des Einsatzes unterschiedlicher Modellierungskonzepte für Unsicherheiten darstellt, wurde bisher nach Wissen der Autoren nicht fokussiert.

\section{SYSTEMATISIERUNG DER UNSICHERHEITEN DER LEERCONTAINERLOGISTIK}

In bisher publizierten mathematischen Optimierungsmodellen für die Leercontainerlogistik, erfolgt oft nur ein Einbezug zweier zentraler Unsicherheiten - zum 
einen des Leercontainerangebots (,supply“), der sich aus dem vorhanden Lagerbestand und den eingehenden Leercontainern zusammensetzt, und zum anderen bzgl. des Leercontainerbedarfs (,demand“), der sich aus der Summe der Leercontainernachfragen durch die Exporteure ergibt (siehe Abbildung 2). Diese Betrachtung erfolgt meist zu allen Planungsperioden für alle relevanten Regionen, Depots, Container-Terminals etc. Die Beschränkung auf diese Arten der unsicheren Einflussfaktoren ist jedoch oft $\mathrm{zu}$ allgemein und spiegelt nicht die gesamten Unsicherheiten in den Planungsprozessen wider. Weiterhin ist eine detailliertere Betrachtung für eine angemessene Ursachenforschung notwendig, die Basis für eine Bewertung geeigneter mathematischer Modellierungsmethoden für die verschiedenen Unsicherheiten im Leercontainermanagement ist. Zur detaillierteren Analyse ist im Folgenden der Kreislauf eines Containers mit Fokus auf die Leerphase näher erläutert.

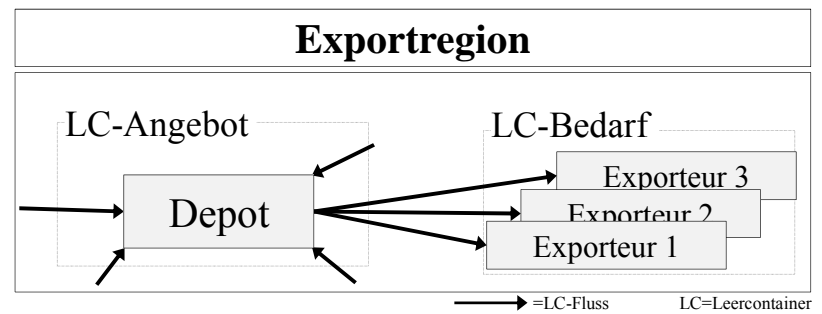

Abbildung 2. Traditionell als unsicher betrachtete Parameter

\subsection{Prozessorientierte SYSTEMATISIERUNG}

Die relevanten Unsicherheiten des Leercontainermanagements lassen sich in drei Kategorien einteilen: Mengen-/ Kapazitäts-, Zeit- und Kostenunsicherheiten. Diese Unsicherheitskategorien erstrecken sich für alle Prozesse über den gesamten Kreislauf eines Containers, der sich aus Last- und Repositionierungsphase zusammensetzt (siehe Abbildung 3).
Nach dem erfolgten Vollcontainertransport wird dem Importeur eine vertraglich vereinbarte Frist zur Rückgabe des Containers zu einem in der Nähe befindlichen Depot gegeben. Importeure benötigen jedoch oft länger zum Entladen und Rücktransport, wodurch die Wiedereinsatzzeit und somit die Planungsgrundlage beeinflusst wird. Ist der Container vom Importeur zurückgekehrt, müssen eventuell Maßnahmen zur Instandhaltung erfolgen, die den Container auf den nächsten Lasteinsatz vorbereiten. Insbesondere bei Lebensmitteltransporten ist teilweise eine Reinigung oder Prüfung der Temperaturregelung notwendig. Je nach Verschmutzungen oder Beschädigungen, die aufgrund der globalen Transporte und häufigen Umschlagprozesse auftreten können, kann die Dauer bis zur Wiedereinsatzfähigkeit variieren. Sobald der Container wieder verfügbar ist, wird er ohne zeitnahen Folgeauftrag in einem Depot zwischengelagert. Die Unsicherheiten im Lagerungsprozess sind mit unsicheren Kosten sowie Maximalbeständen verbunden. Grund ist z. B. die gemeinsame Nutzung von Depots durch mehrere Containerbesitzer.

Ist kein Folgeauftrag in der Region abzusehen, in einer anderen Region jedoch Bedarf vorhanden, wird der Leercontainer repositioniert. Hierfür wird im Falle einer globalen Repositionierung ein Slot auf einem Containerschiff vorgemerkt und der Container zum Containerterminal gebracht. Für den Umschlag auf das Containerschiff existiert jedoch neben schwankenden Umschlagskosten die Unsicherheit der maximalen Umschlagsmenge. Die Reedereien operieren nach dem „Cut \& Run“-Prinzip [DCZ08]. Dies bedeutet, dass Containerschiffe aufgrund vorbestimmter Zeitfenster in den Häfen vor der vollständigen Ausführung geplanter Umschlagsprozesse ablegen. Insbesondere auf Leercontainer wird verzichtet, um eine pünktliche Abfahrt zu sichern. Dies ist eine Ausprägung folgender Philosopie der Reedereien: Vollcontainertransporte generieren direkte Gewinne und besitzen dementsprechend gegenüber Leercontainerprozessen Priorität.

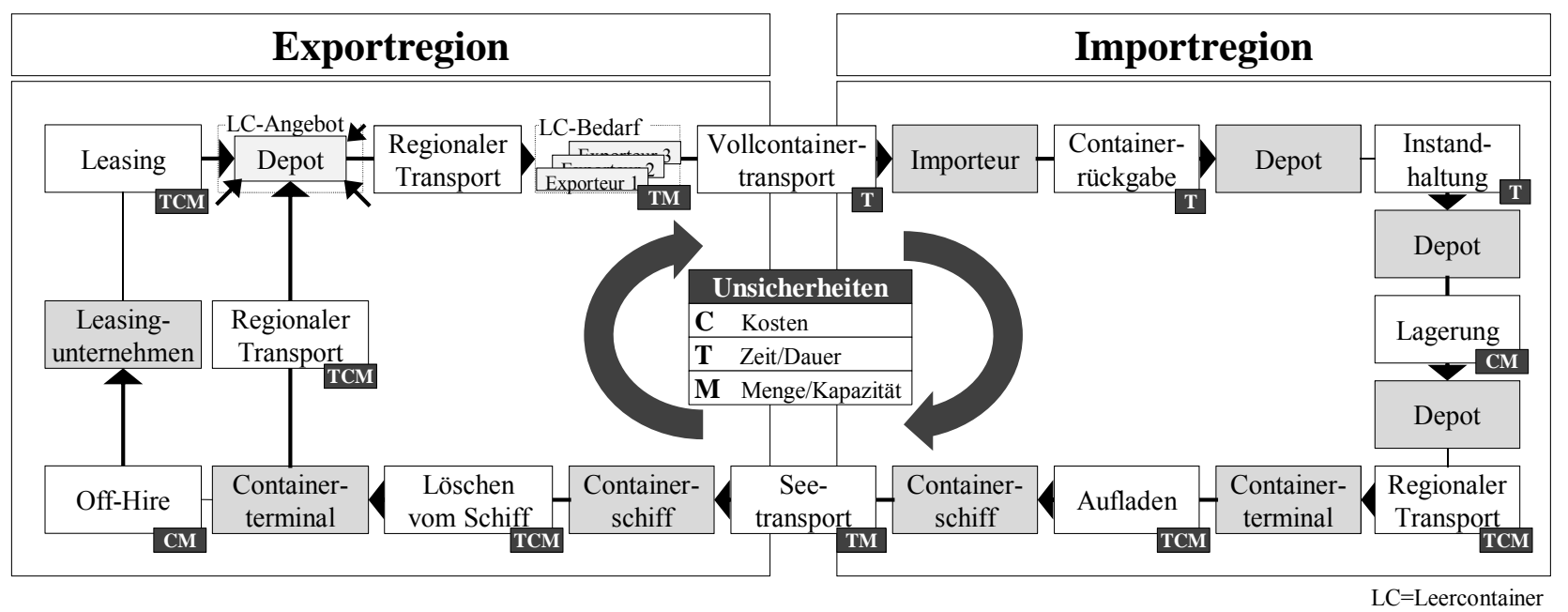

Abbildung 3. Prozessorientierte Systematisierung der Unsicherheiten im Leercontainermanagement 
Nach Verladung des Leercontainers erfolgt der Seetransport. Die Slots für Leercontainer können dabei in Abhängigkeit von den Vollcontainern schwanken. Die Ankunft des Schiffs am Zielhafen ist aufgrund von Einflussfaktoren wie technischen Mängeln, Wetterbedingungen oder auch des „Slow Steaming“-Trends (Schiffe werden bei Bedarf angewiesen, langsamer zu fahren, um Treibstoffkosten zu reduzieren) nicht mit absoluter Gewissheit bekannt. Dennoch folgen Liniendienste den festen Fahrplänen der Reedereien, so dass die Transportdauer in der Regel minimale Abweichungen zeigt. Das Löschen des Containers vom Schiff ist wiederum mit schwankenden Umschlagskosten und einer nicht exakt bekannten Abladezeit verbunden.

Anschließend erfolgt der Transport zu einem Depot, falls noch kein sofortiger Containerbedarf besteht, oder direkt zum Exporteur. Die Kosten, Kapazitäten und Transportdauer des regionalen Transports sind abhängig von den eingesetzten Verkehrsträgern: Binnenschifffahrt, Schienen- und Straßenverkehr. Unwetter, technische Mängel und Unfälle sind nur einige Ursachen für die Entstehung von Unsicherheiten.

Ein für die Containerlogistik relevanter Spezialfall ist der Einsatz von Leasingcontainern. Reedereien sind aufgrund des Wettbewerbsdrucks bestrebt, jeden Auftrag zu erfüllen. Daher wird bei Mangel eigener Container und Unwirtschaftlichkeit des Kaufs neuer Container die Option des Leasings genutzt. Die Anzahl maximal verfügbarer Container sowie anfallende fixe und variable Kosten sind jedoch langfristig nicht exakt bekannt. Für geleaste Container gelten vertraglich vereinbarte Regeln, die unterschiedliche Ausprägungen besitzen können. Für den operativen Planungsprozess sind insbesondere „Short Term“leasing Verträge in die Betrachtung zu integrieren. Bei Anwendung dieser Vertragsform können Leercontainer bereits nach kurzer Zeit zurückgegeben (,Off-Hire“) werden. Allerdings sichern die Leasingunternehmen durch standortabhängige Kosten und maximale Abgabemengen die Rückgabe in Bedarfsregionen ab. Aus diesem Grund sind die Reedereien auch für geleaste Container mit dem Problem der Repositionierung unter unsicheren Einflüssen konfrontiert.

Aus der Vielzahl der Kreisläufe der einzelnen Container setzt sich an der Schnittstelle Depot unter Berücksichtigung verschiedener Containertypen der verfügbare Leercontainerbestand zu einem bestimmten Zeitpunkt zusammen. Der Bedarf an Leercontainern durch potentielle Exporteure ist wiederum durch Einflussfaktoren wie wirtschaftliche oder auch politische Schwankungen nicht als sicher zu betrachten. Dennoch ist der Bedarf bei entsprechender Datenverwaltung wenige Tage vorher mit geringer Abweichung prognostizierbar, da der Großteil der Aufträge früher eingeht und kurzfristige Aufträge in einem gewissen Maße vorhersagbar sind [DCZ08].
Es ist zu bemerken, dass der beschriebene Kreislauf sich nicht auf die Gesamtheit aller möglichen Prozesse bezieht, sondern einen repräsentativen Kreislauf für den regelmäßig auftretenen Fall der globalen Repositionierung darstellt. Auf die detaillierte Erklärung der Prozessabläufe des Vollcontainertransports wurde verzichtet, da diese nicht unmittelbar im Zusammenhang mit den Unsicherheiten des operativen Leercontainermanagements stehen. Weiterhin kann der Kreislauf in Abhängigkeit des Reeders und Praxisfalls variieren. So ist der Prozess der Instandhaltung auch nach dem Seetransport möglich, falls dies z. B. aufgrund von Lohnkostengefällen sinnvoll ist. Individuelle Betrachtungen sind somit notwendig, im Rahmen dieses Beitrags jedoch nicht zielführend.

Ersichtlich wird aus der Beschreibung der Prozesse, wie komplex und unsicher die Planung des Leercontainerangebots ist. Ziel der Reedereien muss die Gleichheit von Angebot und Nachfrage in jeder Region zu jedem Zeitpunkt sein. Dies ist jedoch nur in theoretischen Betrachtungen möglich. Die Berücksichtigung von Unsicherheiten in den Planungsprozessen, die durch mathematische Optimierungsmodelle unterstützt werden, ist daher unerlässlich.

\subsection{ANSATZ ZUR URSACHENANALYSE DER UNSICHERHEITEN}

Nach Systematisierung der Unsicherheiten des Leercontainermanagements sollten Akteure die Ursachen der einzelnen Unsicherheiten adäquat analysieren. Auf diese Weise ist zum einen die Festlegung einer Unsicherheitsart für die Inputparameter der Optimierung möglich und zum anderen der angemessene Umgang der Entscheidungsträger mit dem final gewählten Modellierungskonzept gewährleistet. Beispielsweise kann bei der Anwendung einer Fuzzy-Modellierung mit vagen Angaben die Verbesserung von Expertenaussagen durch Kenntnis der Ursachen für Unsicherheiten erzielt werden.

Für die Ursachen-Wirkungs-Analyse existieren vielfältige Methoden wie Cognitive Maps oder die Fehlerbaumanalyse. Die wohl bekannteste Methode ist das sogenannte Ishikawa-Diagramm. Dieses stellt UrsacheWirkungs-Beziehungen auf mehreren Ebenen dar. Die Ursachen für die Unsicherheiten können auf die oberste Ebene nach dem in Abschnitt 2.1 beschriebenen Ansatz von Zimmermann in Informationsmangel, Informationsüberfluss, Informationskonflikt, Informationsmehrdeutigkeit und Subjektivität differenziert werden. Dieser Ansatz soll im Rahmen dieses Beitrags exemplarisch und ohne Anspruch auf Allgemeingültigkeit und Vollständigkeit für die Unsicherheit über die maximale Umschlagsmenge für Leercontainer auf ein Containerschiff dargestellt werden (siehe Abbildung 4). 


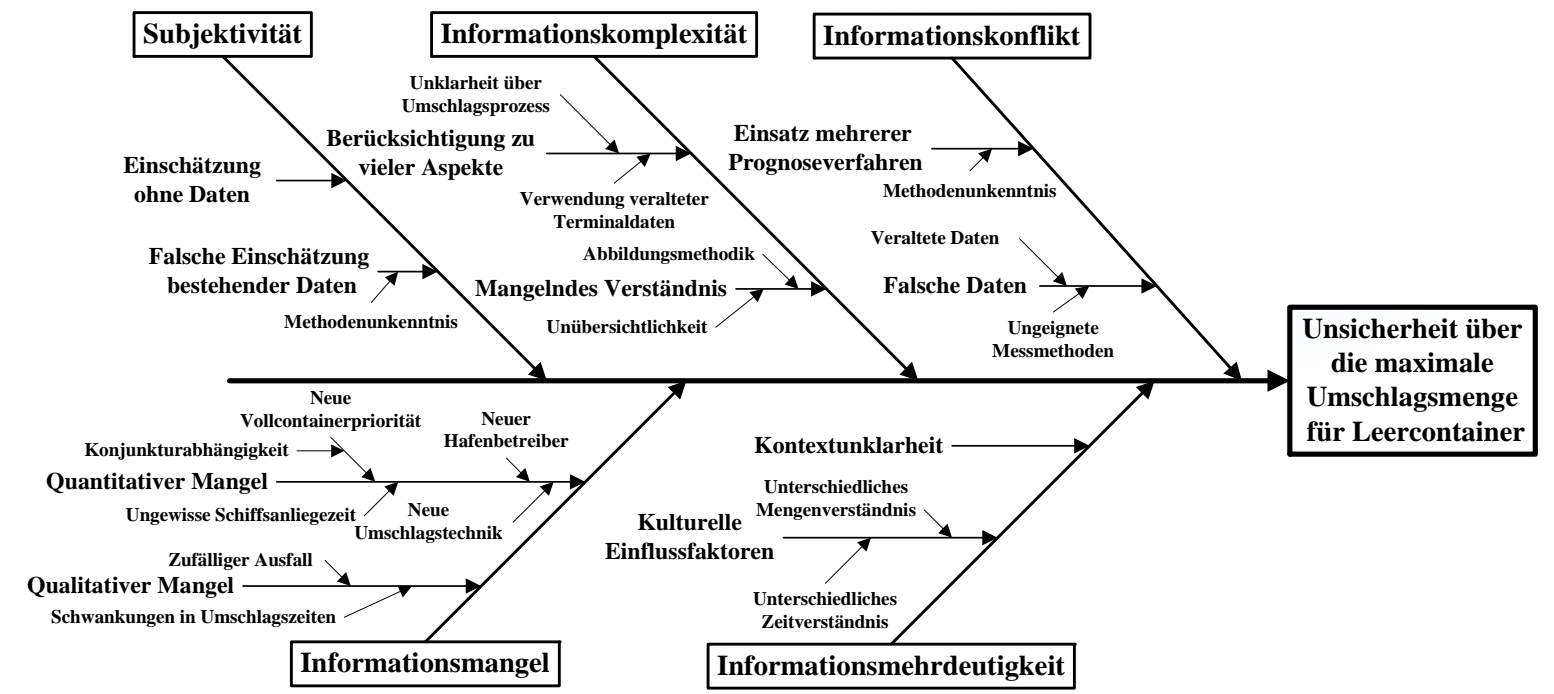

Abbildung 4. Beispiel: Ursachenanalyse für die Unsicherheit der maximalen Umschlagsmenge für Leercontainer

Eine detailliertere und der Praxis gerecht werdende Darstellung ist von oder mit Entscheidungsträgern zu erarbeiten. Weiterhin ist eine fallspezifische Unterscheidung stets notwendig.

\section{FALLBEISPIEL: EINSATZ DES GREY-MODELLIE- RUNGSKONZEPTS FÜR DIE NETZWERKOPTIMIERUNG}

Ein Großteil von veröffentlichten mathematischen Optimierungsmodellen basiert auf einen Raum-ZeitNetzwerkansatz. Dieser Ansatz wird in diesem Kapitel kurz erläutert und anschließend die Modifizierung durch den Einsatz des Grey-Modellierungskonzepts vorgestellt.

\subsection{DER NETZWERKANSATZ}

Ein mathematisches Netzwerk $\mathrm{N}$ ist ein Tupel $\mathrm{N}=(\mathrm{V}, \mathrm{E}, \mathrm{q}, \mathrm{s}, \lambda, \kappa)$ mit einem gerichteten Graphen $(\mathrm{V}, \mathrm{E})$, bestehend aus einer Knotenmenge $\mathrm{V}$ und einer Kantenmenge $\mathrm{E} \subseteq \mathrm{V} \times \mathrm{V}$, sowie zwei ausgezeichneten Knoten $\mathrm{q}, \mathrm{s} \in \mathrm{V}$. Der Knoten q wird als Quelle bezeichnet, $s$ als Senke. Die Kapazitätsfunktionen $\lambda, \kappa: E \rightarrow \mathbb{R}_{+}$ordnen jeder Kante eine untere bzw. obere Kapazität zu. Weiterhin lässt sich das Netzwerk mit einer Kostenfunktion $\gamma: E \rightarrow \mathbb{R}$ erweitern. Mit diesem Ansatz lassen sich Handlungsmöglichkeiten im Leercontainermanagement modellieren.

Alle relevanten Standorte für die Reedereien wie Containerterminals, Depots oder auch anliegende Containerschiffe werden als Knoten modelliert. Diese Knoten werden nun für mehrere Planungsperioden dupliziert. Eine Planungsperiode kann ein festes Zeitintervall (Tag, Stunde etc.) sein oder in der Länge von bestimmten Ereignissen (Schiffsankunft, Auftragseingang etc.) abhängen. Im Anschluss werden die Möglichkeiten für Lagerung und Transport durch Kanten modelliert. Eine Kante zwischen zwei Knoten, die den gleichen Ort repräsentieren, entspricht einer Lagerung. Die obere Kapazität modelliert in diesem Fall den Maximalbestand, die untere
Kapazität den Sicherheitsbestand. Die Kosten der Kante entsprechen den Kosten für die Lagerung eines Containers in einer Periode. Transportmöglichkeiten (hierzu zählt auch der Umschlag) werden durch Kanten repräsentiert, die Knoten verschiedener Standorte verbinden. Kapazitäten und Kosten können analog zur Lagerung modelliert werden. Der Einbezug von Leercontainerbestand und -bedarf kann durch Kanten von der Quelle bzw. zur Senke stattfinden. Ein Beispiel des allgemeinen Raum-ZeitNetzwerk-Ansatzes ist in Abbildung 5 dargestellt.

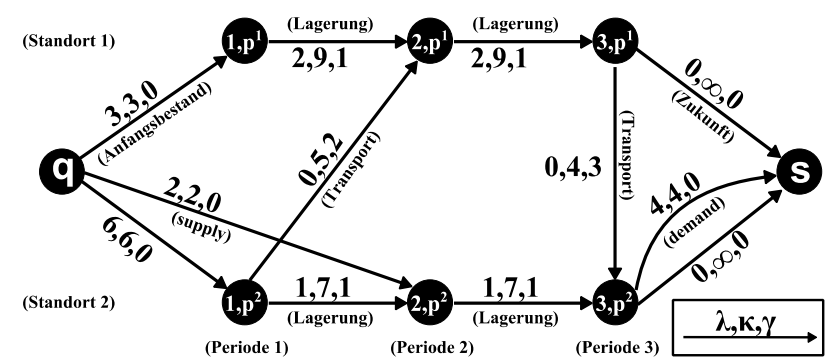

Abbildung 5. Beispiel: Raum-Zeit-Netzwerk-Ansatz

Auf den Kanten kann nun eine Flussfunktion $\varphi: E \rightarrow \mathbb{N}$ definiert werden, die jeder Kante Flusseinheiten, im betrachteten Fall Leercontainer, zuweist. Diese Flussfunktion soll zum einen die Bedingung erfüllen, dass für jeden Knoten im Netzwerk die Menge der Einheiten die „eingehen“ der Menge der Einheiten, die ,ausgehen“ entspricht (Flussbedingung) und dass für jede Kante die untere bzw. obere Kapazität nicht unter-/ überschritten wird (Kapazitätsbedingung). Insgesamt ergibt ein ganzzahliges lineares Optimierungsproblem, bestehend aus einer Zielfunktion der Gesamtkosten Z und den genannten Restriktionen:

$$
\begin{gathered}
\mathrm{Z}=\sum_{\mathrm{e} \in \mathrm{E}} \varphi(\mathrm{e}) \gamma(\mathrm{e}) \rightarrow \min \\
\sum_{\mathrm{u} \in \mathrm{V}: \mathrm{vu} \in \mathrm{E}} \varphi(\mathrm{vu})=\sum_{\mathrm{u} \in \mathrm{V}: \mathrm{uv} \in \mathrm{E}} \varphi(\mathrm{uv}) \quad \forall \mathrm{v} \in \mathrm{V} \backslash\{\mathrm{q}, \mathrm{s}\}
\end{gathered}
$$




$$
\lambda(\mathrm{e}) \leq \varphi(\mathrm{e}) \leq \kappa(\mathrm{e}) \quad \forall \mathrm{e} \in \mathrm{E}
$$

$$
\varphi(\mathrm{e}) \in \mathbb{N} \quad \forall \mathrm{e} \in \mathrm{E}
$$

Dies ist ein sogenanntes Min Cost Flow Problem, für das für $\lambda, \kappa: E \rightarrow \mathbb{N}$ in polynomieller Zeit eine ganzzahlige Lösung, also ein kostenminimaler Fluss, bestimmt werden kann. Die Ganzzahligkeit ist erforderlich, da eine Flusseinheit einen Leercontainer repräsentiert. Für die Betrachtung mehrerer Container ist die Erweiterung zu einem Integer Multicommodity Min Cost Flow Problem notwendig, welches ein NP-vollständiges Problem ist und somit nur mit „hohem“ Aufwand lösbar ist. Auf nähere Erläuterungen dieser Erweiterung und die Einbeziehung weiterer Optionen wie des Leasings oder des Neukaufs, wird im Rahmen dieses Beitrags verzichtet.

\subsection{DiE MODELLIERUNG MIT GREY}

In der Praxis können Kosten oft kurzfristig in einem sicheren Intervall vorhergesagt werden. Der Einsatz von Grey-Zahlen für gewisse Kosten ist daher für mathematische Optimierungsmodelle denkbar. Im Folgenden wird die Integration dieses Konzepts zur Berücksichtigung von Unsicherheiten auf Basis des Netzwerkansatzes veranschaulicht.

Die Kostenfunktion ist folgendermaßen zu modifizieren: $\gamma^{ \pm}: \mathrm{E} \rightarrow\left[\mathrm{g}^{-}, \mathrm{g}^{+}\right]$, wobei die unteren und oberen Intervallgrenzen von den Entscheidungsträgern nach eingehender Analyse der Ursachen für die Unsicherheit festzulegen sind. Zur Vereinfachung der Anwendung in der Praxis ist statt der einzelnen Bestimmung der Intervallgrenzen auch der Einsatz einer zeitabhängigen Funktion vorstellbar, die mit zunehmender Zeit die Intervalle angemessen größer werden lässt. Wachsende Intervallgrößen modellieren somit steigende Unsicherheit bzgl. weiter in der Zukunft liegenden Entwicklungen. Für alle anderen Kanten, die nicht in die Grey-Modellierung einfließen, gilt $\mathrm{g}^{-}=\mathrm{g}^{+}$. Die modifizierte Optimierung besteht nun aus zwei nacheinander auszuführenden Optimierungsproblemen [Ros09]. Zunächst wird die Zielfunktion des Optimierungsproblems aus Abschnitt 5.1 durch folgende ersetzt:

$$
Z^{-}=\sum_{\mathrm{e} \in \mathrm{E}} \varphi^{-}(\mathrm{e}) \gamma^{-}(\mathrm{e}) \rightarrow \min
$$

Die Funktion $\mathrm{Z}^{-}$entspricht den Gesamtkosten für die niedrigsten Kostenausprägungen $\mathrm{y}^{-}$. Die optimale Lösung wird mit $\varphi_{\text {opt }}^{-}$bezeichnet. Im Anschluss findet eine zweite Optimierung statt, in der zum einen die Zielfunktion des ursprünglichen Optimierungsmodells durch $\mathrm{Z}^{+}=\sum_{\mathrm{e} \in \mathrm{E}} \varphi^{+}(\mathrm{e}) \gamma^{+}(\mathrm{e}) \longrightarrow \min$ ersetzt wird $\left(\mathrm{y}^{+}\right.$entspricht der maximalen Kostenausprägung) und zum anderen zusätzlich die sogenannte Interaktionsrestriktion gefordert wird:

$$
\varphi^{+}(\mathrm{e}) \geq \varphi_{\text {opt }}^{-}(\mathrm{e}) \quad \forall \mathrm{e} \in \mathrm{E}
$$

Die optimalen Lösungen der beiden Probleme stellen dem Entscheidungsträger stabile und zulässige Bereiche für die Zielfunktion und die Entscheidungsvariablen zur Verfügung. Die Wahl einer Lösung aus dem Bereich $\left[\varphi_{\text {opt }}^{-}, \varphi_{\text {opt }}^{+}\right]$stellt sicher, dass sich die Gesamtkosten im Bereich $\left[\mathrm{Z}^{-}, \mathrm{Z}^{+}\right]$befinden [Ros09].

\section{FAZIT UND AUSBLICK}

Die optimale Gestaltung von Leercontainerflüssen und -beständen ist aufgrund globaler Handelsungleichgewichte ein zentrales Problem der maritimen Containerlogistik. In der Vergangenheit wurden zahlreiche mathematische Optimierungsmodelle entwickelt, um die Entscheidungsträger in ihren Handlungen zu unterstützen. Die Präsenz von Unsicherheiten in den Planungsprozessen sowie das komplexe System der globalen Containerlogistik erschweren jedoch den Einsatz der Optimierungsmodelle in der Praxis. In diesem Beitrag wurden im speziellen Kontext des Leercontainermanagements Unsicherheiten systematisiert, entsprechende Modellierungskonzepte vorgestellt und Ansätze zur Anwendung dieser aufgezeigt. Die Ergebnisse sollen Basis für die verbesserte Planung von Leercontainerprozessen in der Praxis sowie der Realität angepassten mathematischen Optimierungsmodellen sein. In Zukunft soll die Analyse weiterer Modellierungsmethoden für Unsicherheiten erfolgen und die Möglichkeit des gemischten Einsatzes in Optimierungsmodellen untersucht werden. Zur Unterstützung der Analysen soll die Zusammenarbeit mit Praxispartnern weitergeführt werden.

\section{LITERATUR}

Bean, W. L.; Joubert, J. W.: Planning empty container relocations under uncertainty. In: South African Journal of Industrial Engineering, 21(1), S. 113122,2010

Borgelt, C.; Kruse, R.: Unsicherheit und Vagheit: Begriffe, Methoden, Forschungsthemen. In: Künstliche Intelligenz, Themenheft Unsicherheit und Vagheit, 15(3), S. 5-8, 2001

[CC98] Cheung, R. K.; Chen, C. Y.: A two stage stochastic network model and solution methods for dynamic empty container allocation problem. In: Transportation Science, 32(2), S. 142-162, 1998 
[CGD93] Crainic, T. G.; Gendreau, M.; Dejax, P.: Dynamic and stochastic models for the allocation of empty containers. In: Operations Research, 41(1), S. 102-126, 1993

[CGT10] Chou, C. C.; Gou, R. H.; Tsai, C. L.; Tsou, M. C.; Wong, C. P.; Yu, H. L.: Application of a mixed fuzzy decision making and optimization programming model to the empty container allocation. In: Elsevier; Applied Soft Computing, 10, S. 1071-1079, 2010

[DCZ08] Di Francesco, M.; Crainic, T. G.; Zuddas, P.: The effect of multi-scenario policies on empty container repositioning under uncertainty and data shortage. Interuniversity research centre on enterprise networks, logistics and transportation, 2008

[EKP76] Ermol'ev, Yu. M.; Krivets, T. A.; Petukhov, S.: Planning of shipping empty seaborne containers. In: Cybernetics, 12(4), S. 644-646, 1976

[EMS05] Erera, A. L.; Morales J. C.; Savelsbergh M. W. P.: Global intermodal tankcontainer management for the chemical industry. In: Transportation Research Part E, 41(6), S. 551-566, 2005

[EMS07] Erera, A. L.; Morales, J. C.; Savelsbergh, M. W. P.: Robust Optimization for Empty Repositioning Problems. In: The Supply Chain and Logistics Institute School of Industrial and Systems Engineering Georgia Institute of Technology Atlanta, GA 30332-0205, September 28, 2007

[ENW12] Epstein, R.; Neely, A.; Weintraub, A.; Valenzuela, F.; Hurtado, S.; Gonzales, G.; Beiza, A.; Naveas, M.; Infante, F.; Alarcon, F.; Angulo, G.; Berner, C.; Catalan, J.; Gonzales, C., Yung, D.: A Strategic empty container logistics optimization in a Major Shipping Company. In: Interfaces; 42(1), S. $5-16,2012$

[Gle76] Shafer, G.: A Mathematical Theory of Evidence, Princeton University Press, 1976

[HJO10] Helton, J. C.; Johnson, J. D.; Oberkampf, W. L.; Sallaberry, C. J.: Representation of analysis results involving aleatory and epistemic uncertainty. In: International Journal of General Systems, 39(6), S. 605-646, 2010

[Hüt12] Hüttmann, B.: Aspekte zur Optimierung und ganzheitliche Bewertung der Leercontainerlogistik. BSL Transportation Consultants GmbH \& Co. KG; Vortrag im Rahmen der Veranstaltung „Logistik trifft Wissenschaft" der Logistikinitiative Hamburg, 3. Mai 2012

[LLC12] Long, Y.; Lee, L. H.; Chew, E. P.: The sample average approximation method for empty container repositioning with uncertainties. In: European Journal of Operational Research 222, S. 65-75, 2012

[OZF05] Olivo, A.; Zuddas, P.; Di Francesco, M.; Manca, A.: An operational model for empty container management. In: Maritime Economics and Logistics, 7(3), S. 199-222, 2005

[Paw82] Pawlak, Z.: Rough sets. In: International Journal of Parallel Programming, 11(5), S. 341-356, 1982

[Ros09] Rosenberg, D. E.: Shades of grey: A critical review of grey-number optimization. In: Engineering Optimization, 41(6), S. 573-592, 2009

[SK95] Shen, W. S.; Khoong, C. M.: A DSS for empty container distribution planning. In: Information Technology Institute. Decision Support Systems, 15(1), S. 7582, 1995

[Voj10] Vojdani, N.: Optimierung der Leercontainerbereitstellung in Seehäfen mittels Container-Pooling. 10. Rostocker Logistik Forum 2010, Rostock, 23.-24. Juni 2010

[Zad65] Zadeh, L. A.: Fuzzy sets. In: Information and Control, 8 (3), S. 338-353, 1965

[Zad78] Zadeh, L. A.: Fuzzy Sets as the Basis for a Theory of Possibility. In: Fuzzy Sets and Systems 1, S. 3-28, 1978

[Zim00] Zimmermann, H. J.: An applicationoriented view of modeling uncertainty; In: European Journal of Operational Research 122, S. 190-198, 2000 